\title{
TITLE: CHEBYSHEV MOMENT PROBLEMS: MAXIMUM ENTROPY AND KERNEL PLYNOMIAL METHODS
}

\author{
Atuhors R. N. Silver \\ H. Roeder \\ A. F. Voter \\ J. D. Kress
}

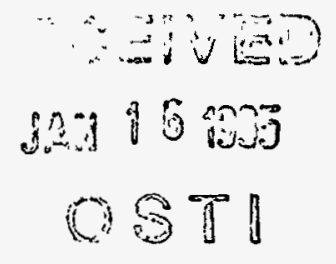

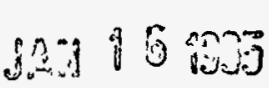

(1)
SUBMTTED TO:

\section{DISCLAIMER}

This report was prepared as an account of work sponsored by an agency of the United States Government. Neither the United States Government nor any agency thereof, nor any of their employees, makes any warranty, express or implied, or assumes any legal liability or responsibility for the accuracy, completeness, or usefulness of any information, apparatus, product, or process disclosed, or represents that its use would not infringe privately owned rights. Reference herein to any specific commercial product, process, or service by trade name, trademark, manufacturer, or otherwise does not necessarily constitute or imply its endorsement, recommendation, or favoring by the United States Government or any agency thereof. The views and opinions of authors expressed herein do not necessarily state or reflect those of the United States Government or any agency thereof. 


\title{
CHEBYSHEV MOMENT PROBLEMS:
}

MAXIMUM ENTROPY AND KERNEL POLYNOMIAL METHODS

\author{
R. N. SILVER, H. ROEDER, A. F. VOTER AND J. D. KRESS \\ Theoretical Division \\ MS B262 Los Alamos National Laboratory \\ Los Alamos, NM $87545^{\dagger}$
}

\begin{abstract}
Two Chebyshev recursion methods are presented for calculations with very large sparse Hamiltonians, the kernel polynomial method (KPM) and the maximum entropy method (MEM). They are applicable to physical properties involving large numbers of eigenstates such as densities of states, spectral functions, thermodynamics, total energies for Monte Carlo simulations and forces for tight binding molecular dynamics. This paper emphasizes efficient algorithms.
\end{abstract}

Key words: computational physics, convex optimization, density of states, electronic structure, kernel polynomial method, large sparse matrices, moment problems, maximum entropy.

\section{Introduction}

Many computational physics problems involve calculations with very large sparse Hamiltonian matrices. Finding all eigenvectors and eigenvalues requires cpu time scaling as $O\left(N^{3}\right)$ and memory scaling as $O\left(N^{2}\right)$, which is impractical. For ground or isolated eigenstates the preferred method is Lanczos diagonalization, which uses only matrix-vector multiply operations and requires cpu and memory scaling as $O(N)$. But new $O(N)$ methods are needed for properties involving many eigenstates such as the density of states (DOS) and spectral functions, and for quantities that can be derived from DOS such as thermodynamics, total energies for electronic structure and forces for molecular dynamics and Monte Carlo simulations. In such applications, limited energy resolution and statistical accuracy are often acceptable provided the uncertainties can be quantified. Maximum entropy (MEM) $[1,2]$ has been a popular approach to such problems, usually fitting power moments of a DOS or spectral function. However, the non-linear convex optimiza-

\footnotetext{
t to appear in Maximum Entropy and Bayesian Methods 1995, eds. K. Hanson and R. N. Silver, Kluwer Academic Press; e-mail: rns@loke.lanl.gov
} 
tion algorithms required to find MEM solutions may be difficult to implement for large numbers of power moments and for singular structures in DOS.

Calculation of Chebyshev moments is much less sensitive than power moments to the limitations of machine precision. Chebyshev series are also preferred because of their isomorphism to Fourier series which enables use of advanced methods of Fourier analysis such as FFT's, Gibbs damping, etc. This paper discusses the generation of Chebyshev moment data, describes a simple linear Chebyshev approximation termed the kernel polynomial method (KPM) [3,4], and then it presents an efficient MEM algorithm [5].

Consider the DOS as representative of the properties of interest. The first step is to scale the Hamiltonian, $\mathbf{H}=a \mathbf{X}+b$ such that all eigenvalues $x_{n}$ of $\mathbf{X}$ lie between -1 and +1 . The DOS is then

$$
D(x)=\frac{1}{N} \sum_{n=1}^{N} \delta\left(x-x_{n}\right) .
$$

The data about $D(x)$ consists of Chebyshev moments,

$$
\mu_{m}=\operatorname{Tr}\left\{T_{m}(\mathrm{X})\right\}=\int_{-1}^{1} T_{m}(x) D(x) d x
$$

Calculation of moments uses Chebyshev recursion,

$$
T_{m+1}(\mathrm{X})=2 \mathrm{X} T_{m}(\mathrm{X})-T_{m-1}(\mathrm{X}),
$$

requiring the same optimized matrix-vector-multiply algorithm in Lanczos methods. Unlike Lanczos recursion, Chebyshev recursions are numerically stable to arbitrarily large numbers of moments without any need for expensive reorthogonalization. Exact evaluation of $M$ moments uses cpu time $\propto O\left(N^{2} M\right)$. A stochastic method [3], scaling as $O\left(N M N_{r}\right)$, uses estimators

$$
\widehat{\mu}_{m} \approx \frac{1}{N_{r}} \sum_{r}<r\left|T_{m}(\mathrm{X})\right| r>,
$$

where $\mid r>$ are $N_{r}$ Gaussian random vectors. Such data have calculable statistical variance proportional to $\left(N N_{r}\right)^{-1}$. If the Hamiltonian has only local off-diagonal elements, as in tight-binding Hamiltonians, a non-stochastic locally truncated approximation to the Hamiltonian $\mathrm{H}_{i}$ may be adequate [6]. The estimator,

$$
\widehat{\mu}_{m} \approx \sum_{i}<i\left|T_{m}\left(\mathbf{X}_{i}\right)\right| i>
$$

generates data with a systematic error determined by the truncation range. Cpu scales as $O(N M J)$, where $J$ is the average number of states in the truncation range. Exact moment derivatives (which are related to forces) can also be calculated.

\section{The Kernel Polynomial Method}

Cpu time and memory limit the number of moments $M$ and their statistical and systematic errors. Given such limited data, KPM and MEM are two ways to estimate DOS. KPM $[3,4]$ provides a linear Chebyshev approximation to a DOS with 
a uniform resolution in $\phi=\cos ^{-1}(x)$. It is based on an exact moment expansion,

$$
D(x)=\frac{1}{\pi \sqrt{1-x^{2}}}\left[\mu_{0}+2 \sum_{m=1}^{\infty} \mu_{m} T_{m}(x)\right] \text {. }
$$

The KPM truncates this expansion at $M$ moments and introduces a factor $g_{m}^{M}$ to damp the Gibbs phenomenon,

$$
D_{K}(x)=\frac{1}{\pi \sqrt{1-x^{2}}}\left[\mu_{0}+2 \sum_{m=1}^{M} \mu_{m} g_{m}^{M} T_{m}(x)\right] .
$$

Let $D(\phi) \equiv \sin (\phi) D(X)$ and $T_{m}(x)=\cos (m \phi)$. Then $D_{K}(\phi)$ is both a simple convolution and a truncated Fourier series,

$$
D_{K}(\phi)=\int_{0}^{2 \pi} \delta_{K}\left(\phi-\phi_{o}\right) D\left(\phi_{o}\right) d \phi_{\circ} \quad ; \quad \delta_{K}(\phi)=\frac{1}{2 \pi}\left[g_{0}+2 \sum_{m=1}^{M} g_{m}^{M} \cos (m \phi)\right] \text {. }
$$

The "kernel" $\delta_{K}(\phi)$ is a $2 \pi$-periodic polynomial approximation to a Dirac delta function, analogous to the resolution function of a spectrometer. Resolution is uniform in $\phi$ with width $\Delta \phi \propto M^{-1}$. If $g_{m}^{M}=1$, at large $|\phi|$ the kernel is oscillatory with period $\Delta \phi=\pi / M$ within an envelope function decreasing slowly as $1 / \phi^{2}$. The result is the Gibbs phenomenon of a lack of uniform convergence at singular structures in DOS. An optimal $g_{m}^{M}$ can be determined variationally by requiring the kernel to be a polynomial of degree $M$, strictly positive, normalized and have minimal variance in $\phi$ [7]. Specifically, by the Fejer-Riesz theorem

$$
\delta_{K}(\phi)=\frac{1}{2 \pi}\left|\sum_{\nu=0}^{M} a_{\nu} e^{i \nu \phi}\right|^{2} \quad ; \quad g_{m}^{M}=\sum_{\nu=0}^{M-m} a_{\nu} a_{\nu+m} .
$$

To obtain the best energy resolution minimize the variance,

$$
\Delta \phi^{2} \equiv \int_{-\pi}^{\pi} \phi^{2} \delta_{K}(\phi) d \phi \simeq \int_{-\pi}^{\pi}(2-2 \cos (\phi)) \delta_{K}(\phi) d \phi=2 g_{0}-2 g_{1},
$$

subject to a normalization constraint, $\int_{-\pi}^{\pi} \delta_{K}(\phi) d \phi=1$, or equivalently $g_{0}^{M}=1$. That is, the variational problem,

$$
Q=g_{1}-\lambda g_{0}=\sum_{\nu=0}^{M-1} a_{\nu} a_{\nu+1}-\lambda \sum_{\nu=0}^{M} a_{\nu} a_{\nu}
$$

results in

$$
\frac{\delta Q}{\delta a_{\nu}}=0 \quad \Rightarrow a_{\nu+2}-2 \lambda a_{\nu+1}+a_{\nu}=0 .
$$

The solution to Eq. (12) is

$$
a_{\nu}=\frac{U_{\nu}(\lambda)}{\sqrt{\sum_{\nu=0}^{M} U_{\nu}^{2}(\lambda)}} ; \quad U_{\nu}(\lambda)=\frac{\sin \left((\nu+1) \phi_{\lambda}\right)}{\sin \left(\phi_{\lambda}\right)} ; \cos \left(\phi_{\lambda}\right)=\lambda,
$$




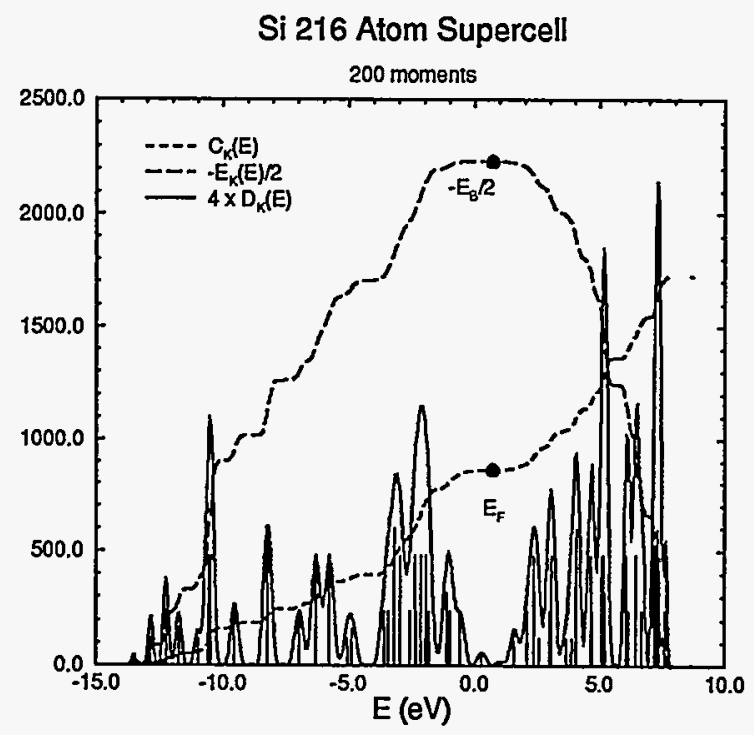

Figure 1. KPM DOS and band energy calculation for Si 216 atom supercell.

where the $U_{\nu}$ are Chebyshev polynomials of the second kind. The same kernel is also obtained by minimizing the uniform norm [8]. Its envelope function decreases exponentially at large $|\phi|$.

Figure 1 illustrates the application of KPM to the electronic structure of a 216 atom Si supercell using a tight binding Hamiltonian [7] based on the parameterization of Goodwin, et al. [9]. This system is small enough to be exactly diagonalized. Vertical lines are at the energies of the exact eigenstates and their height is proportional to their degeneracy. The solid line is the KPM approximation to the DOS obtained for 200 Chebyshev moments. A Fermi energy $E_{F}$ is the energy at which the cumulative DOS $C_{K}(E)$ equals the number of electrons. The total band energy $E_{B}$ is then the cumulative energy $E_{K}(E)$ at $E_{F}$. For band energies KPM converges $\propto M^{-2}$ reaching $10^{-5}$ relative accuracy at about $M \approx 150$.

KPM can be applied to other properties such as spectral functions [7],

$$
A(\omega)=\lim _{\eta \rightarrow 0+} \frac{1}{\pi} \operatorname{Im}\left\{\left\langle\Psi_{0}\left|\mathbf{O}^{\dagger} \frac{1}{\omega-\mathbf{H}-i \eta} \mathrm{O}\right| \Psi_{0}\right\rangle\right\},
$$

where $\mathrm{O}$ is an operator. KPM approximations use moments $\mu_{m}^{\mathrm{O}}=\left\langle\Psi_{0}\left|\mathrm{O}^{\dagger} T_{m}(\mathrm{X}) \mathrm{O}\right| \Psi_{0}\right\rangle$.

Applications to thermodynamics use a rapidly converging Fourier-Bessel expansion of the partition function [3],

$$
Z=e^{-\beta b}\left[I_{0}(\beta a)+2 \sum_{m=1}^{\infty} I_{m}(\beta a) \mu_{m}\right] \text {. }
$$


The $I_{m}(\beta a)$ are modified Bessel functions. The partition function involves integral rather than pointwise convergence, so the optimal choice is $g_{m}^{M}=1$.

\section{The Maximum Entropy Method}

MEM uses the same Chebyshev moment data as KPM. The entropy,

$$
S \equiv \int_{0}^{\pi}\left[D(\phi)-D_{o}(\phi)-D(\phi) \ln \left(\frac{D(\phi)}{D_{o}(\phi)}\right)\right] d \phi .
$$

Here $D_{o}(\phi)$ is a default model for the DOS in the absence of data. Consider first the case where the data are subject to Gaussian independent statistical noise,

$$
\widehat{\mu}_{m}=\mu_{m}+\eta_{m} ; \mathbf{E} \eta_{m}=0 ; \quad \mathbf{E} \eta_{m} \eta_{m^{\prime}}=\sigma_{m}^{2} \delta_{m m^{\prime}} .
$$

(E denotes the statistical expectation value of the random variable that follows

it.) In case the data are exact, $\sigma_{m}$ represents the numerical precision required of the MEM fit to the data. The primal optimization problem is to maximize entropy as a function of $D(\phi)$ constrained by the known moments. That is, maximize

$$
Q_{p} \equiv S-\frac{\chi^{2}}{2 \alpha} ; \chi^{2}=\sum_{m=0}^{M}\left(\frac{\widehat{\mu}_{m}-\mu_{m}}{\sigma_{m}}\right)^{2} \text {. }
$$

The statistical regularization parameter $\alpha$ sets a balance between the fit, measured by $\chi^{2}$, and an information measure, $-S$, of distance between the inferred $D(\phi)$ and the default model $D_{o}(\phi)$. (Alternatively, $1 / \alpha$ is a Lagrange multiplier.) The $m=0$ term is included to constrain normalization, $\widehat{\mu}_{0}=1$. Taking the limit $\sigma_{0} \rightarrow 0$ strictly enforces normalization.

Our MEM algorithm consists of three nested loops: iterations in $\alpha$, until a stopping criterion is reached; at each $\alpha$, Newton-Raphson iterations of a dual optimization problem to solve for the MEM $D(\phi)$; at each $\alpha$ and MEM $D(\phi)$ conjugate gradient iterations to apply the Hessian onto a vector.

Popular stopping criteria for $\alpha$ are $\chi^{2}=M$ and $\chi^{2}-2 \alpha S=M$, although many other criteria are discussed in the literature. However, the algorithm for finding the MEM $D(\phi)$ tends to be unstable if initiated at such small $\alpha$. Instead, start at large $\alpha^{1} \approx \chi_{o}^{2}$, and use $D_{o}(\phi)$ to initiate the optimization of $D^{1}(\phi)$. Progress down in $\alpha$ such that $\alpha^{k+1}=\alpha^{k} / 2$. If this is unstable, halve the step down in $\alpha$ repeatedly until stability is reached. At each $\alpha$, use $D^{k}(\phi)$ as the starting point for the optimization of $D^{k+1}(\phi)$. Once the stopping criterion is passed, perform a golden search for the optimal $\alpha$.

In the case of exact moment data, set $\sigma_{m}$ to the numerical precision required, which can be very small. In our applications to electronic structure, errors of one part in $10^{5}$ or smaller were used. Iterate $\alpha \rightarrow 0$ until the entropy $S$ saturates at an $\alpha$-independent value.

Given an $\alpha$, a variety of algorithms have been developed to find MEM solutions $[10,11]$. The primal optimization problem maximizes $Q_{p}$ as a function of $D(\phi)$,

$$
\frac{\delta Q_{p}}{\delta D(\phi)}=-\ln \left(\frac{D(\phi)}{D_{o}(\phi)}\right)+\sum_{m=0}^{M} \frac{\widehat{\mu}_{m}-\mu_{m}}{\alpha \sigma_{m}^{2}} \cos (m \phi)=0
$$


which has a unique solution. Define parameters $\vec{\lambda}$ by

$$
\widehat{\mu}_{m}-\mu_{m}+\alpha \sigma_{m}^{2} \lambda_{m}=0 \text {. }
$$

Then the $D(\phi)$ satisfying Eq. (19) is

$$
D(\phi)=D_{o}(\phi) \exp \left(-\sum_{m=0}^{M} \lambda_{m} \cos (m \phi)\right) .
$$

This form is also obtained by maximizing entropy subject to Lagrange contraints on moments with Lagrange multipliers $\vec{\lambda}$.

However, a dual optimization problem as a function of the $M$ Lagrange multipliers [12] solves the same problem, and it is more stable numerically than the primal problem. The $\vec{\lambda}$ of the dual problem vary more slowly than the $D(\phi)$, and they are a finite rather than a continuous set of variables. The quantity,

$$
Q_{d} \equiv \ln \left(\int_{0}^{\pi} D(\phi) d \phi\right)+\sum_{m=0}^{M}\left[\widehat{\mu}_{m} \lambda_{m}+\frac{\alpha \sigma_{m}^{2} \lambda_{m}^{2}}{2}\right],
$$

is maximized as a function of the $\vec{\lambda}$ when Eq. (20) is satisfied. Away from the maximum, define

$$
\frac{\delta Q_{d}}{\delta \lambda_{m}} \equiv \xi_{m} \equiv \widehat{\mu}_{m}-\mu_{m}+\alpha \sigma_{m}^{2} \lambda_{m}
$$

Then,

$$
Q_{d}=Q_{p}+\sum_{m=0}^{M} \frac{\xi_{m}^{2}}{2 \alpha \sigma_{m}^{2}}
$$

The Hessian of the dual problem is a positive definite $M \times M$ matrix and a simple function of the moments,

$$
H_{m m^{\prime}} \equiv \frac{\partial^{2} Q_{d}}{\partial \lambda_{m} \partial \lambda_{m}^{\prime}}=\frac{\mu_{m+m^{\prime}}+\mu_{\left|m-m^{\prime}\right|}}{2}+\alpha \sigma_{m}^{2} \delta_{m m^{\prime}}
$$

A solution to Eq. (20) may be found by Newton-Raphson iteration. Beginning with some starting $\vec{\lambda}^{0}$, the $n+1^{\prime}$ th step is

$$
\vec{\lambda}^{n+1}=\vec{\lambda}^{n}-\mathbf{H}_{n}^{-1} \vec{\xi}^{n}
$$

The quantity, $\mathrm{H}^{-1} \vec{\xi}$, may be calculated, e.g., by conjugate gradients. In view of Eq. (24), converging bounds at the $n^{\prime}$ th iteration are $Q_{d}^{n} \geq Q^{\infty} \geq Q_{p}^{n}$ where $Q^{\infty} \equiv \lim _{n \rightarrow \infty}\left\{Q_{d}^{n}, Q_{p}^{n}\right\}$. This provides stopping criteria for the iteration.

For electronic structure applications, high numerical precision (e.g. $\approx 10^{-6}$ ) is needed for accurate energy derivatives. Careful attention to how the MEM algorithms are discretized then becomes very important. Practical fast Fourier transform (FFT) algorithms calculate the $\mu_{m}=\int_{0}^{\pi} \cos (m \phi) D(\phi) d \phi$ by sampling the domain $0 \leq \phi \leq \pi$ at a discrete set of $N_{p}$ equally spaced points. The Shannon 

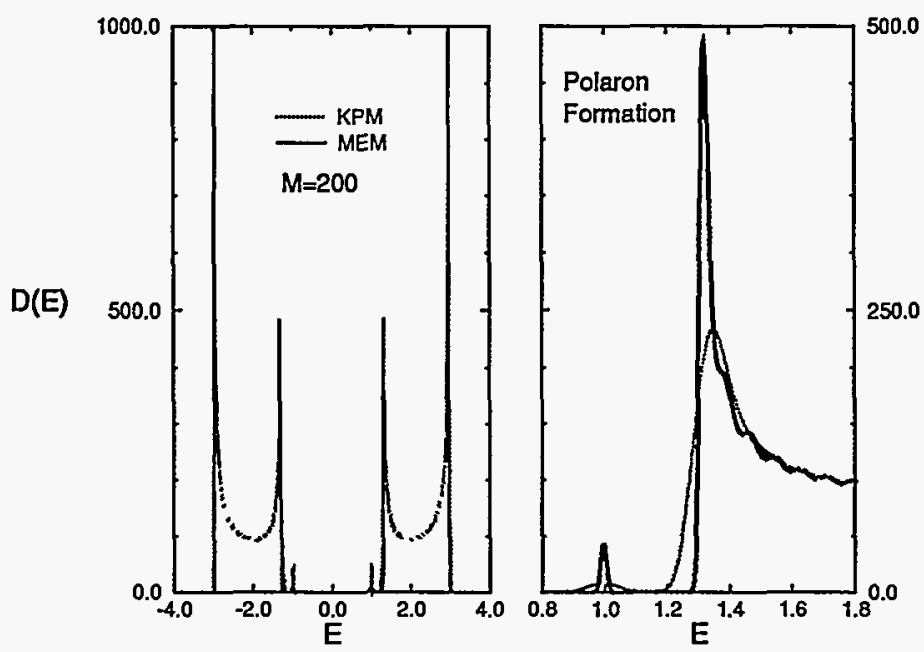

Figure 2. Comparison of KPM and MEM for the DOS of a polaron formation problem using 200 moments.

sampling theorem says that such naive discretization corresponds to representing a DOS in a $N_{p}$-order truncated Chebyshev series. In effect, MEM is used to infer an $N_{p}$-order Chebyshev approximation from knowledge of $M$ true moments.

But typical DOS contain singular structures such as $\delta$-functions, van Hove singularities, band edges, etc. These structures are properly described by an infinite order Chebyshev expansion. As discussed previously for the KPM, abrupt truncation of a Chebyshev expansion at $N_{p}$ terms results in the Gibbs phenomenon; i.e. singular structures in the true DOS at $\phi_{0}$ induce oscillations in Chebyshev approximated DOS of period $\Delta \phi=\pi / N_{p}$ with an envelope function decreasing slowly as $1 /\left(\phi-\phi_{o}\right)^{2}$. While the moment data satisfy the Hausdorff conditions for the existence of a positive solution [1], the added requirement that the solution be an $N_{p}$-order Chebyshev series is stronger. For the exact moment problem, the $\alpha$-iteration may have difficulty forcing $\chi^{2} \rightarrow 0$ and saturating the entropy $S$.

Fortunately, the kernel polynomial method (KPM) provides a solution to this discretization problem. In the MEM problem, replace the $M$ Chebyshev moment data $\widehat{\mu}_{m}$ by modified moments $\widehat{\mu}_{m} g_{m}^{N_{p}}$, where the $g_{m}^{N_{p}}$ are the Gibbs damping factors in Eq. (9). In other words, change the goal of the MEM algorithm to the inference of a $N_{p}$-order KPM approximation to the DOS. Iteration toward $\chi^{2} \rightarrow 0$ and saturation of $S$ becomes easy.

By choosing $N_{p} \gg M$, MEM can achieve significant resolution enhancements over KPM from the same $M$ moments. In tests with tight binding Hamiltonians for the electronic structure of $\mathrm{Si}$ [5], band energies converge approximately 4 times faster with MEM than with KPM. For the example in Fig. 1, MEM reaches $10^{-5}$ 
accuracy at $M \approx 35$. Setting $N_{p} \geq 4 M$ is adequate to achieve this gain. The cpu time required by MEM scales as $O\left(M^{2}\right)$, and it is negligible compared to the cpu time required to generate the moment data. Use of MEM cuts the overall cpu requirements by at least a factor of 4 over KPM. Isolated features in DOS, such as individual states and band edges, may converge even faster.

Figure 2 compares MEM and KPM for the DOS of a 1D polaron formation problem [5]. The Hamiltonian consists of an electron placed into a 10,000 atom chain with a Peierls distortion, which is then allowed to relax resulting in the polaron state at $E=1.0$. MEM achieves dramatically better energy resolution than KPM for isolated states and band edges, but it tends to "ring" (or oscillate) in smooth positive regions of a DOS when singular structures, such as Van Hove singularities, are nearby. For such regions of a spectrum, MEM may converge more slowly than KPM. A solution to the ubiquitous MEM ringing problem most likely will require a modification to the entropy functional to include local smoothness constraints [13].

\section{References}

1. L. R. Mead and N. Papanicolaou, "Maximum entropy in the problem of moments," J. Math. Phys., 25, p. 2404, 1984.

2. D. A. Drabold and O. F. Sankey, "Maximum entropy approach to linear scaling in the electronic structure problem," Phys. Rev. Letts., 70, p. 3631, 1993.

3. R. N. Silver and H. Roeder, "Density of states of megadimensional hamiltonian matrices," Int. J. Mod. Phys. C, 5, pp. 735-753, 1994.

4. L. W. Wang, "Calculating the density of states and optical-absorption spectra for large quantum systems by the plane wave moments method," Physical Review B, 49, pp. 10154$10158,1994$.

5. R. N. Silver and H. Roeder, "Maximum entropy algorithms for chebyshev moment problems," J. Comp. Phys., to appear 1995.

6. A. F. Voter, J. D. Kress, and R. N. Silver, "Linear scaling tight binding from a truncated moments approach," submitted to Physical Review $B, 1995$.

7. R. N. Silver, H. Roeder, A. F. Voter, and J. D. Kress, "Kernel polynomial approximations for densities of states and spectral functions," J. Comp. Phys., to appear 1995.

8. J. D. Jackson, Theory of Approximation, Cambridge University Press, 1930.

9. L. Goodwin, A. J. Skinner, and D. G. Pettifor, "Generating transferable tight binding parameters: Application to silicon," Europhysics Letters, 9, pp. 701-706, 1989.

10. I. Turek, "A maximum entropy approach to the density of states within the recursion method," J. Phys. C: Solid State Physics, 21, pp. 3251-3260, 1988.

11. J. Skilling, "Bayesian numerical analysis," in Physics 8 Probability: Essays in honor of Edwin T. Jaynes, J. W. T. Grandy and P. W. Milonni, eds., pp. 207-221, Cambridge University Press, 1993.

12. C. Auyeng and R. M. Mersereau, "A dual approach to signal restoration," in Digital Image Restoration, A. K. Katsaggelos, ed., pp. 21-55, Springer, 1991.

13. R. N. Silver and H. F. Martz, "Applications of quantum entropy to statistics," in 1994 Proceedings of the Statistical Computing Section, pp. 61-70, American Statistical Association, 1994. 